"This accepted author manuscript is copyrighted and published by Elsevier. It is posted here by agreement between Elsevier and MTA. The definitive version of the text was subsequently published in [Journal of Plant Physiology 171 (2014) 1256-1266, doi:10.1016/j.jplph.2014.04.013]. Available under license CC-BY-NCND."

\title{
A morpho-physiological approach differentiates bread wheatcultivars of contrasting tolerance under cyclic water stress
}

\author{
Katalin Jäger ${ }^{\mathrm{a}}$, Attila Fábián ${ }^{\mathrm{a}}$, Gabriella Eitel ${ }^{\mathrm{a}}$, László Szabób ${ }^{\mathrm{b}}$, Csilla Deák ${ }^{\mathrm{c}}$, \\ Beáta Barnabás ${ }^{\mathrm{a}}$, István Papp ${ }^{\mathrm{c}, *}$ \\ ${ }^{a}$ Agricultural Institute, Centre for Agricultural Research, Hungarian Academy of Sciences, Brunszvik u. 2, 2462 Martonvásár, Hungary ${ }^{b}$ \\ Department of Functional and Structural Materials, Institute of Materials and Environmental Chemistry, Chemical Research Center, \\ Hungarian Academy of Sciences, Pusztaszeri út 59-67, 1025 Budapest, Hungary \\ ${ }^{c}$ Department of Plant Physiology and Plant Biochemistry, Faculty of Horticultural Science, Corvinus University of Budapest, Villányi út 29-43, \\ 1118 Budapest, Hungary
}

\begin{abstract}
Leaf micromorphological traits and some physiological parameters with potential relevance to drought tolerance mechanisms were investigated in four selected winter wheat varieties. Plants were subjected to two cycles of drought treatment at anthesis. Yield components confirmed contrasting drought-sensitive and -tolerant behavior of the genotypes. Drought tolerance was associated with small flag leaf surfaces and less frequent occurrence of stomata. Substantial variation of leaf cuticular thickness was found among the cultivars. Thin cuticle coincided with drought sensitivity and correlated with a high rate of darkadapted water loss from leaves. Unlike in Arabidopsis, thickening of the cuticular matrix in response to water deprivation did not occur. Water stress induced epicuticular wax crystal depositions preferentially on the abaxial leaf surfaces. According to microscopy and electrolyte leakage measurements from leaf tissues, membrane integrity was lost earlier or to a higher extent in sensitive than in tolerant genotypes. Cellular damage and a decline of relative water content of leaves in sensitive cultivars became distinctive during the second cycle of water deprivation. Our results indicate strong variation of traits with potential contribution to the complex phenotype of drought tolerance in wheat genotypes. The maintained membrane integrity and relative water content values during repeated water limited periods were found to correlate with drought tolerance in the selection of cultivars investigated.
\end{abstract}

\section{Introduction}

Common wheat (Triticum aestivum L.) is one of the most important staple crops with potential sensitivity to water stress, especially in the reproductive stage. Drought tolerance of wheat is a complex trait with multifactorial determination, depending on interactions of genotypes and environmental conditions (Araus et al., 2002; Szü cs et al., 2010).

In addition to stomatal transpiration (Sirichandra et al., 2009), the cuticle contributes significantly to water loss, especially under water limited stress conditions (Riederer and Schreiber, 2001). Micromorphological features and properties of this layer may have an impact on water loss and drought tolerance. The cuticle of cereals was found to be relevant in this respect (Rawson and Clarke, 1988),

\footnotetext{
* Corresponding author. Tel.: +361482 6095; fax: +3612096388

E-mail address: istvan.papp2@uni-corvinus.hu (I. Papp).
}

which was also confirmed by recent research efforts (González and Ayerbe, 2010; Wang et al., 2012a,b).

Water limitation and a range of other abiotic stresses may result in oxidative burden to plants (Mittler, 2002). Diverse antioxidant defense responses are mobilized in order to prevent excessive accumulation of potentially harmful reactive oxygen species (ROS) (Gill and Tuteja, 2010). Sustained drought stress may result in abundant ROS production that cannot be balanced by the antioxidant system, leading to deleterious oxidative events including membrane damage and cell death (Cruz de Carvalho, 2008). Membrane injury has been shown to occur during severe water stress in wheat and has been associated with drought sensitivity and poor induction of antioxidant defense responses (Khanna-Chopra and Selote, 2007). Structural damage of organelles has been found to be associated with drought sensitivity in some other crops as well (Zhang et al., 2010).

This study investigates drought-sensitive Cappelle Desprez, GK Élet and tolerant Plainsman V, Mv Emese winter wheat cultivars subjected to two cycles of drought during anthesis and at early 


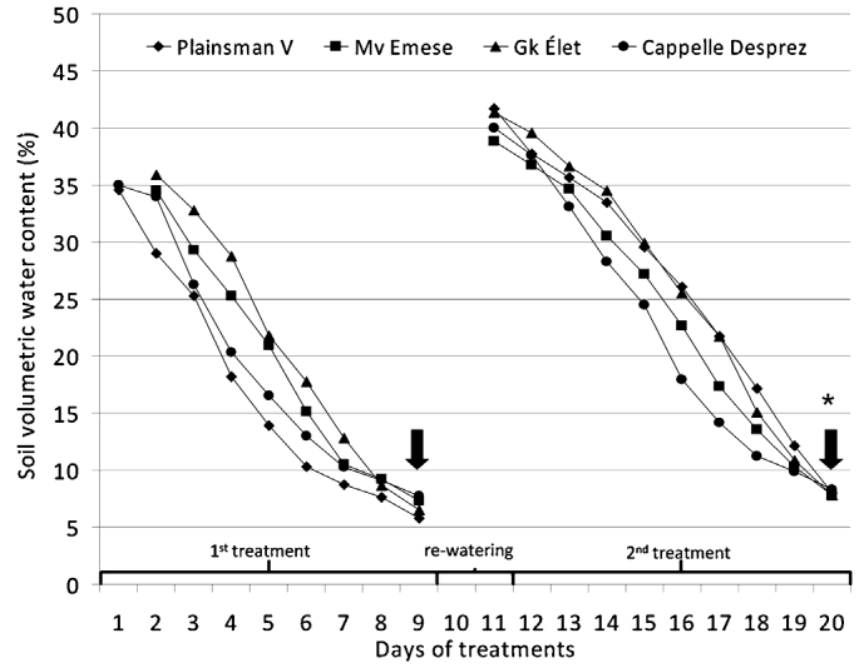

Fig. 1. Volumetric water content of soil during the two drought cycles of the experiment. Arrows indicate sampling dates for relative water content (RWC), relative water loss (RWL) and ion leakage measurements; asterisk marks samplings for light and electron microscopic studies.

stages of kernel development and grain filling. Cycles of water deprivation are thought to approximate natural conditions better than single treatments (Izanloo et al., 2008). Yield parameters as well as micromorphological traits with potential significance to drought hardiness were explored. We aimed to reveal differences of epidermal structures among the genotypes, with an emphasis on stomata and the cuticle. Cellular integrity of mesophyll cells and the rate of electrolyte leakage from flag leaves were also investigated during cyclic drought stress periods.

\section{Materials and methods}

Plant material and stress conditions

Wheat (Triticum aestivum L.) plants were grown in soilsand-peat mixture $(3: 1: 1, \mathrm{v} / \mathrm{v} / \mathrm{v})$ after 7 weeks of vernalization at a temperature of $4{ }^{\circ} \mathrm{C}$, in phytotron chambers (Conviron, Winnipeg, Canada) using the spring climatic program T1 (Tischner et al., 1997). The first cycle of total water withholding started 3 days prior to anthesis (GS58; Zadoks et al., 1974) and lasted for 9 days in Mv Emese and GK Élet, and 10 days in Plainsman V and Cappelle Desprez. After the first treatment, plants were re-watered $(150 \mathrm{~mL})$ for two days and subjected to the second run of water deprivation for 10 days. The volumetric water content of the soil dropped below $10 \%$ (Fig. 1) at the end of both treatments. Along with the treated plants, a control group was grown with normal water supply. Twenty plants of each genotype and treatment were grown to full maturity at a final max/min temperature of $32 / 18{ }^{\circ} \mathrm{C}$ and yield components were determined. All experiments were repeated at least two times during 2010 and 2011.

\section{Determination of soil and flag leaf water content}

Soil volumetric water content (VWC) was measured using an HH2 moisture meter (Delta-T Devices Ltd., Cambridge, UK) at field capacity and during treatments. Relative water content (RWC) of leaves was determined on whole flag leaves of three plants per genotype and treatment at the end of both drying episodes using fresh weight $(\mathrm{FW})$ at excision, saturated weight (SW) after $24 \mathrm{~h}$ rehydration on distilled water at $4{ }^{\circ} \mathrm{C}$ in the dark, and dry weight (DW) after oven drying for $48 \mathrm{~h}$ at $80{ }^{\circ} \mathrm{C}$. The leaf RWC (\%) was calculated using the following equation: $\operatorname{RWC}(\%)=\frac{\mathrm{FW}-\mathrm{DW}}{\mathrm{SW}-\mathrm{DW}} \times 100$
Dark adapted water loss from detached leaves

Water loss (WL) was determined from whole flag leaves of five well watered plants per genotype at 5 days after anthesis (DAA) in Mv Emese, GK Élet and at 6 DAA in Plainsman V and Cappelle Desprez cultivars. All steps of the experiment preceding dry weight determination were conducted under dark conditions. Excised leaf blades were brought to $100 \%$ relative water content by placing leaves on the surface of moistened filter paper overnight. Fresh weight $(\mathrm{FW})$ was measured at full saturation and after drying for 20, $40,60,80,100,120,180,240,300,360,420 \mathrm{~min}$ at $30^{\circ} \mathrm{C}$. Dry weight of flag leaves was determined after drying for $24 \mathrm{~h}$ at $80{ }^{\circ} \mathrm{C}$. The leaf $\mathrm{WL}\left(\mathrm{gh}^{-1} \mathrm{~g}^{-1} \mathrm{DW}\right)$ was calculated using the following equation (Ristic and Jenks, 2002):

Leaf water loss $=\frac{[(\mathrm{FWTx}-\mathrm{FWTx}+1) \times 60]}{[\mathrm{DW} \times(\mathrm{Tx}+1-\mathrm{Tx})]}$, where FWTx is flag leaf fresh weight at time Tx, FWTx +1 is flag leaf fresh weight at time $\mathrm{Tx}+1$, DW is flag leaf dry weight, Tx is time (min) when FWTx was determined, and $\mathrm{Tx}+1$ is time (min) when FWTx +1 was determined. Data obtained from five plants were averaged and used for statistical analysis. Determination of ion leakage

Measurement of conductivity was carried out according to Murray et al. (1989). Six cm long segments were cut from the middle part of flag leaves $(n=12$ per genotype and treatment). Each piece was placed in a test tube filled with $10 \mathrm{~mL}$ of deionizer water and agitated overnight at room temperature under dark conditions. Next day the actual conductivity of the solution was measured using a Mikro KKT Conductometer (Budapest, Hungary). This was followed by treatment of the samples at $121{ }^{\circ} \mathrm{C}, 103.4 \mathrm{kPa}$ for $40 \mathrm{~min}$ to disrupt all membranes in the cells. Conductivity was measured again and the values obtained were assumed to represent complete (100\%) electrolyte leakage.

Yield components

Main spikes of control and drought-stressed plants $(n=20$ per treatment) for each genotype were harvested at full maturity. Total floret number per spike, seed number per spike, yield per spike and total plant weight were determined. Fertility and harvest index were calculated from these data.

\section{Light microscopy}

Flag leaves of main tillers were collected from 6 plants per genotype and treatment at the end of the second exposure to water stress. According to our preliminary studies, trichome density widely differed with location both on adaxial and abaxial leaf surfaces. In order to eliminate data distortion, 3-3 stereomicroscopic images were taken from basal, middle and top portions of each leaf before the clearing procedure. After area measurements and stereomicroscopic determination of trichome density $\left(\mathrm{n} \mathrm{mm} \mathrm{m}^{-2}\right)$, leaves were cleared overnight in a solution containing 95\% ethanol (41\%), chloroform (21\%), lactic acid (17\%), phenol $(21 \%)$ and chloral hydrate $(3.63 \mathrm{M})$, washed, and stored in $50 \%$ ethanol. Cleared epidermal peels of each flag leaf were manually dissected from the middle part of the specimen as a thin transparent film using thin forceps, flattened on the surface of distilled water, mounted on microscope slides in 50\% glycerol and examined with an Olympus B51 microscope (Olympus, Tokyo, Japan). Ten micrographs were taken of each of the peels dissected from both abaxial and adaxial side of the leaves at $200 \times$ magnification. The computeraided determination of epidermal parameters was performed using $\mathrm{Cell}^{\mathrm{P}}$ image analysis software (Olympus, Tokyo, Japan). Pavement cell density (PF $\left.\left[\mathrm{n} \mathrm{mm}^{-2}\right]\right)$, silica cell density, stomatal frequency (SF $\left.\left[\mathrm{n} \mathrm{mm}^{-2}\right]\right)$, and guard cell length $(\hat{\mathrm{l}})$ were measured. The 
area-independent stomatal index was calculated from PF and SF, as a percentage, according to the formula (Salisbury, 1927):

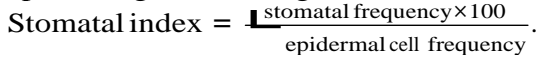

The central regions of control and stress-treated flag leaves $\left(\mathrm{n}=5\right.$ per treatment and genotype) were cut into $1 \mathrm{~mm}^{2}$ parts at the end of the second drought episode, fixed in $50 \mathrm{mM} \mathrm{Na}$ cacodylate buffer ( $\mathrm{pH}$ 7.2) containing $2.5 \%$ glutaraldehyde (v/v) and $4 \%$ formaldehyde $(\mathrm{w} / \mathrm{v})$, washed, dehydrated in a series of ethanol and gradually infiltrated with epoxy resin according to

Spurr (1969). The resin was polymerized at $65{ }^{\circ} \mathrm{C}$ for $48 \mathrm{~h}$. Semi-thin sections (1 $\mathrm{Lm}$ ) were cut using an Ultracut-E microtome (ReichertJung, Heidelberg, Germany) and stained with periodic acid-Schiff (PAS) and Coomassie Brilliant Blue for polysaccharides and proteins, respectively. The stained sections were mounted in Depex Mounting Medium (EMS, Fort Washington, PA) and examined using a BX51 light microscope (Olympus, Tokyo, Japan).

\section{Transmission electron microscopy}

The middle parts of 6 flag leaves of wheat (per genotype and per treatment) were cut into $1 \mathrm{~mm}^{2}$ pieces and fixed for $4 \mathrm{~h}$ at room temperature in primary fixative containing $2.5 \%(\mathrm{v} / \mathrm{v})$ glutaraldehyde in $0.05 \mathrm{M}$ Na-cacodylate buffer, $\mathrm{pH} 7.2$ (CB). After fixation, the samples were washed in $\mathrm{CB}$ and post-fixed for $3 \mathrm{~h}$ at $4{ }^{\circ} \mathrm{C}$ in $1 \%(\mathrm{w} / \mathrm{v})$ osmium tetroxide in $0.05 \mathrm{M} \mathrm{CB}$. After washing in $\mathrm{CB}$, the samples were dehydrated through a gradient series of ethanol, infiltrated with Spurrs embedding medium (Spurr, 1969) according to the manufacturer's instructions (Sigma-Aldrich, St. Louis, MO), and polymerized for $48 \mathrm{~h}$ at $60{ }^{\circ} \mathrm{C}$. Ultrathin sections were cut using an Ultracut E microtome and mounted on Formvar-coated (SPI-Chem, West Chester, PA) 100-mesh nickel grids stained with $3 \%(\mathrm{w} / \mathrm{v})$ aqueous uranyl acetate and $0.08 \%(\mathrm{w} / \mathrm{v})$ lead citrate. The sections were examined using a Zeiss EM-910 electron microscope (Wetzlar, Germany) at $80 \mathrm{kV}$.

Scanning electron microscopy

For scanning electron microscopy, $1 \mathrm{~mm}^{2}$ parts of 6 flag leaves of wheat (per genotype and per treatment) were fixed for $3 \mathrm{~h}$ at $4{ }^{\circ} \mathrm{C}$ in $2.8 \%$ glutaraldehyde in $0.1 \mathrm{M}$ HEPES buffer $(\mathrm{pH} 7.2)$, washed in HEPES buffer and post-fixed for $3 \mathrm{~h}$ in aqueous $1 \% \mathrm{OsO}_{4}$. After rinsing in HEPES buffer, the tissues were dehydrated in a graded ethanol series, critical point dried (CPD 030, BAL-TEC GmbH, Balzers, Liechtenstein), mounted on nickel grids and coated with gold (SCD 005, BAL-TEC GmbH, Balzers, Liechtenstein). Leaf surfaces were examined using a Zeiss EM-910 electron microscope at $80 \mathrm{kV}$.

Statistical analysis

All data were pooled means from the replicates and were statistically evaluated using ANOVA (SSPS for Windows, version 10.0).

\section{Results}

Effects of drought stress on water content of the soil, flag leaf RWC and yield components

Withholding of water was maintained until the volumetric water content (VWC) of the soil dropped below $10 \%$ at the end of both soil drying episodes. Changes of VWC are shown in Fig. 1. Curves were aligned by matching the time points of the first rewatering. The first drought cycle took one day longer for Plainsman $\mathrm{V}$ and Cappelle Desprez compared to the other two cultivars. In most experiments, Plainsman V and Cappelle Desprez showed faster initial loss of water from the pots, but minor variability was
Table 1

Relative water content (means \pm standard deviations) of control and droughtstressed flag leaves excised at the end of treatments. Note that the genotype had no effect on water loss during the first water withholding, except Cappelle Desprez. Means superscripted by the same letter are not significantly different at the $\mathrm{P} \leq 0.05$ level of probability.

\begin{tabular}{lcccc}
\hline Genotype & Control 1 & Treated 1 & Control 2 & Treated 2 \\
\hline Plainsman V & $86.0^{\mathrm{a}} \pm 4.1$ & $61.4^{\mathrm{b}} \pm 5.5$ & $91.5^{\mathrm{a}} \pm 2.3$ & $48.9^{\mathrm{cd}} \pm 3.7$ \\
Mv Emese & $86.5^{\mathrm{a}} \pm 0.5$ & $55.2^{\mathrm{b}} \pm 2.9$ & $91.5^{\mathrm{a}} \pm 2.6$ & $45.1^{\mathrm{d}} \pm 2.9$ \\
GK Élet & $86.4^{\mathrm{a}} \pm 3.3$ & $55.3^{\mathrm{b}} \pm 1.7$ & $92.4^{\mathrm{a}} \pm 1.8$ & $33.6^{\mathrm{e}} \pm 5.1$ \\
Cappelle Desprez & $91.5^{\mathrm{a}} \pm 1.2$ & $51.3^{\mathrm{c}} \pm 1.3$ & $90.8^{\mathrm{a}} \pm 5.6$ & $33.8^{\mathrm{e}} \pm 3.5$ \\
\hline
\end{tabular}

also observed among repetitions. Compared to the controls, the RWC of treated flag leaves significantly decreased after both exposures to total water withholding (Table 1). No significant difference was observed in the decline of flag leaf RWC among varieties after the first treatment, with the exception of Cappelle Desprez, which displayed a significant drop of RWC already by this time (Table 1).
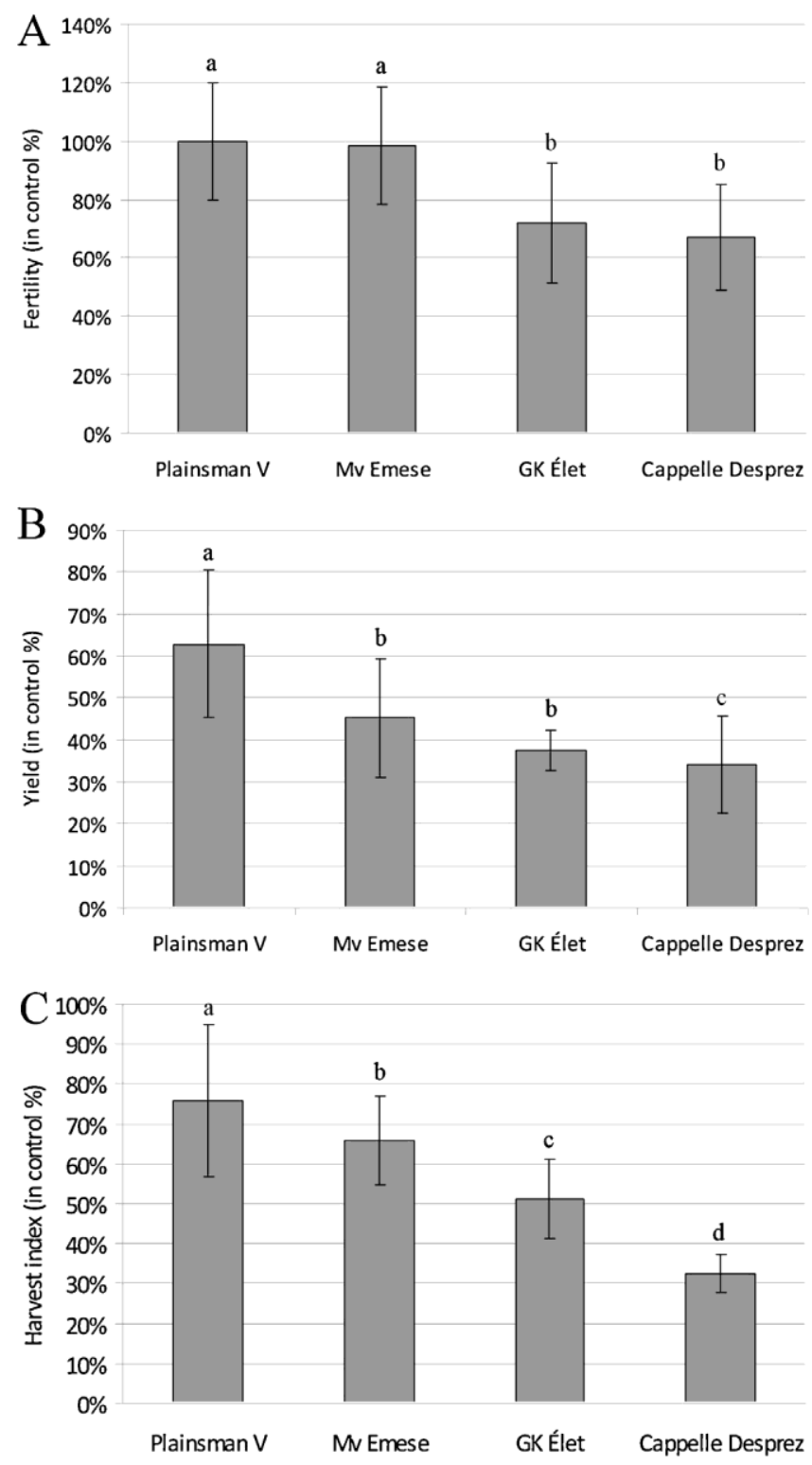

Fig. 2. Fertility (a), yield (b) and harvest index (c) of drought-stressed Plainsman V, Mv Emese, GK Élet and Cappelle Desprez plants compared to their controls. In each graph, columns superscripted by the same letter are not significantly different at the $\mathrm{P} \leq 0.005$ level of probability. 
According to RWCs calculated after the second drying episode, the genotypes studied could be divided into two subgroups: water retention ability of leaf tissues from Plainsman V and Mv Emese was greater than that of GK Élet and Cappelle Desprez.

Water deficit had no effect on the fertility of Plainsman V and Mv Emese genotypes. The same parameter of GK Élet and Cappelle Desprez, however, dropped significantly $(\mathrm{P} \leq 0.005)$ compared to their controls and to the treated plants of the two above-mentioned cultivars (Fig. 2A). The yield of drought-stressed Plainsman V plants was the highest, showing a significant $32.2 \%$ decrease $(\mathrm{P} \leq 0.0005)$ compared to the untreated plants of the same cultivar (Fig. 2B). Compared to their controls, drought-stressed Mv Emese, GK Élet and Cappelle Desprez showed significant ( $\mathrm{P} \leq 0.0005)$ 54.9\%, 62.5\% and $65.9 \%$ drops in yield, respectively. The harvest index (HI) was significantly reduced by withholding water in all genotypes (Fig. 2C). The harvest index decreased by 24.3, 35.6, 48.6 and 67.56 in Plainsman V, Mv Emese, GK Élet and Cappelle Desprez cultivars, respectively.

Micromorphological features of wheat leaves exposed to water stress

As flag leaves of the plants were fully developed 3 days prior to anthesis, stress by water withholding had no effect on the size or the frequency of various cell types located in the epidermis. No difference was found in leaf thickness among the genotypes (data not shown). The flag leaf area of the Plainsman V cultivar was the smallest and that of Cappelle Desprez was the largest (Fig. 3A). The total number of the epidermal cells per $\mathrm{mm}^{2}$ was the lowest in Plainsman V and the highest in Cappelle Desprez (Fig. 3B). Among all cell-types investigated there was a significant difference $(\mathrm{P} \leq 0.0005)$ in cell density between the abaxial and adaxial leaf surfaces (Fig. 3C-F). The frequency of long pavement cells (Fig. 3C), trichomes (Fig. 3E) and stomata (Fig. 3F) was significantly higher on the adaxial surface of the leaves in all genotypes. However, the frequency of short pavement cells was significantly higher on the adaxial surface of the leaves (Fig. 3D). According to our observations, round-shaped short pavement cells seem to be somewhat abaxial counterparts of adaxial trichomes. The extremely high standard deviation of trichome density was due to irregular trichome distribution through the leaf surface with the tendency of higher number on the top and base of adaxial and abaxial surfaces, respectively (data not shown). The number of stomata was the lowest in Plainsman V and the highest in Cappelle Desprez on both leaf surfaces (Fig. 3F). Stomatal indices of leaves were significantly higher on abaxial surfaces (Fig. 3G). Compared to Plainsman V, Cappelle Desprez showed significantly lower stomatal indices (Fig. 3G). The length of guard cells was similar both in abaxial and adaxial epidermises (Fig. 3H). Compared to Cappelle Desprez, the guard cells of Plainsman V were significantly larger ( $\mathrm{P} \leq 0.0005)$. Genotypes Mv Emese and GK Élet showed intermediate values in all epidermal parameters counted or measured (Fig. 3). The thickness and ultrastructure of the cuticles were observed by transmission electron microscopy. There was no difference between the cuticlar thickness of abaxial and adaxial leaf surfaces in the same genotype (Supplementary Figs. 1A and B). The cuticle of GK Élet was the thickest $(113-119 \mathrm{~nm})$ and that of Cappelle Desprez was the thinnest (71-76 nm) (Fig. 4; Supplementary Fig. 1). Drought treatments did not result in any significant changes of cuticular thickness (Supplementary Fig. 1). SEM was applied to visualize drought-induced wax formation on leaf surfaces. There was an orientation-dependent disparity of wax morphology between the two sides of the leaves observed in all but one of the genotypes. Platelet-like epicuticular wax crystals were present on the adaxial faces of flag leaves in all of the cultivars (Fig. 5), and also on the abaxial side of $\mathrm{Mv}$ Emese leaves (Fig. 6C). Longer, tubular structures were found on the abaxial leaf faces of the other genotypes (Fig. 6), which formed a dense mesh in Cappelle Desprez consisting of very long filaments (Fig. 6G and H). Heavier loads of the abaxial tubular wax were evident on the drought-treated samples (Fig. 6B, D, F and H).

Light microscopy was used on leaf cross sections in order to reveal any cellular damage associated with drought treatments. There was no difference observed in the flag leaf structure of the four genotypes (Fig. 7A-D). Our results showed the appearance of shrunken mesophyll cells and disintegrated chloroplasts in flag leaves of treated GK Élet and Cappelle Desprez plants (Fig. 7G and $\mathrm{H})$. At the same time, little cellular damage was observed in leaf tissues of the other two cultivars (Fig. 7E and F).

Dark adapted water loss and electrolyte leakage from leaf tissues

Water loss rates of dark-adapted flag leaf samples from nontreated plants were measured in order to estimate residual transpiration. The results showed a higher rate of water loss from leaves of Cappelle Desprez in comparison with all other genotypes at 5-6 days after pollination (Fig. 8). Ion leakage was measured after the first and second drought episodes in leaves of all cultivars. After the first treatment, only cells of Cappelle Desprez leaves suffered substantial loss of electrolytes (Fig. 9). During the second cycle of water stress, electrolyte leakage was maintained from Cappelle Desprez tissues, while the other sensitive genotype, Élet, also reached a high level of conductivity. Leaves of Mv Emese showed immediate values, while cells of Plainsman V released a remarkably low level of electrolytes throughout the experiment.

\section{Discussion}

Four selected winter wheat cultivars were investigated with presumed contrasting behavior under water limiting conditions (Guóth et al., 2009; Sečenji et al., 2010; Fábián et al., 2011). In addition to two Hungarian cultivars (Mv Emese - tolerant, GK Élet - sensitive), the selection contained two well-known standard genotypes (Plainsman V - tolerant, Cappelle Desprez - sensitive). In order to characterize the behavior of the cultivars under the experimental conditions, applied yield components were determined. Our results verified differences among the cultivars, with drought hardiness decreasing in the following order: Plainsman V $>$ Mv Emese $>$ GK Élet $>$ Cappelle Desprez (Fig. 2). This confirms the classification of the genotypes into two sub-groups. Based on responses to application of two drought cycles at anthesis and early kernel development, Plainsman V and Mv Emese were designated as drought-tolerant, while GK Élet and Cappelle Desprez were considered drought-sensitive. Similarly to our previous findings (Fábián et al., 2011), reduction in fertility in GK Élet and Cappelle Desprez and severe decreases in yield and the harvest index in all genotypes indicated sensitivity of the fertilization process and early stages of kernel development (i.e. cellularization of the free-nuclear syncytium, deposition of A-type starch granules and initiation of B-type starch granules) to drought stress.

In a search for traits relevant to drought tolerance, micromorphological features of flag leaves were investigated (Biswal and Kohli, 2013). The number of pavement cells, trichomes and stomata as well as stomatal indices were measured or calculated. Similarly to the findings of Percival (1921) brist-like wheat trichomes were more frequent on the adaxial surface of leaves in all genotypes. Drought-tolerant cultivars showed less frequent occurrences of stomata on smaller flag leaf surfaces (Fig. 3). Guard cells of the stomata were significantly larger in the tolerant genotypes (Fig. 3). An inverse correlation between size and density of stomata has been also noted by Doheny-Adams et al. (2012) in Arabidopsis. In the same study, a less adverse effect of reduced water 

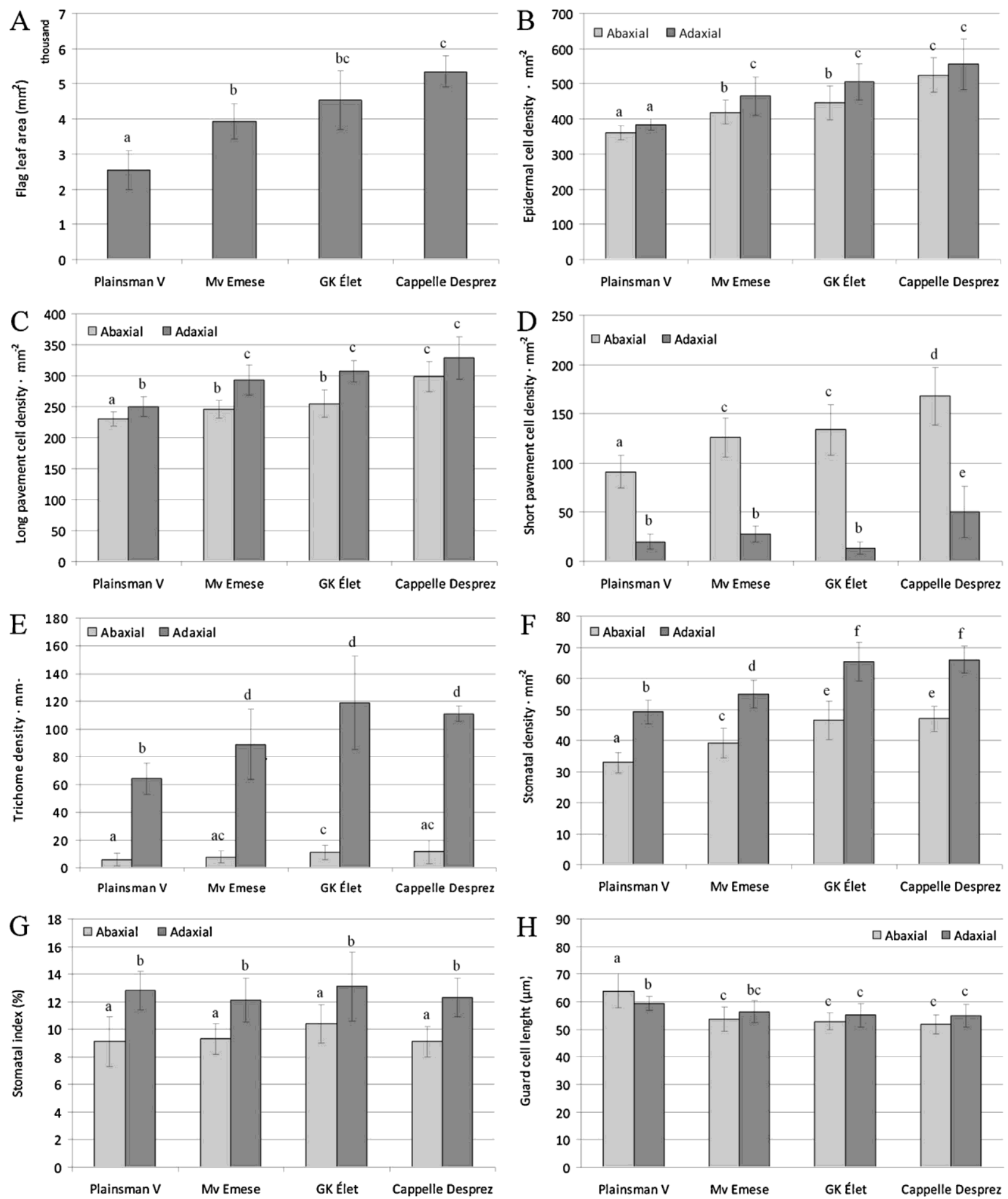

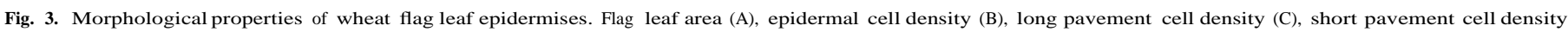

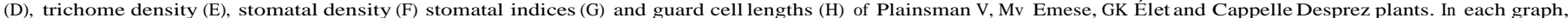
columns superscripted by the same letter are not significantly different minimum at the $\mathrm{P} \leq 0.005$ level of probability.

availability was observed on plants with lower stomatal density, which corresponds well with our results.

A specific ultrastructural feature of shoot morphology is cuticular architecture, with an obvious relevance to water relations. According to the work of Rawson and Clarke (1988), a substantial portion of total water loss from wheat leaves is peristomal under drought conditions. The significance of residual transpiration (RT) with respect to yield is more controversial. González and Ayerbe (2010) found a negative correlation between RT and yield, which emphasizes the importance of water permeance of the cuticle under water stress. Other studies, however, have come to a different conclusion (Clarke et al., 1991). A more direct transgenic approach concerning the relevance of cuticular transpiration by Wang and co-workers $(2012 \mathrm{a}, \mathrm{b})$ has led to the conclusion that decreased water permeability of the cuticle led to improved drought tolerance of rice. In the model plant Arabidopsis, an increased amount of cuticular material of the ABA oversensitive cbp20 mutant has been associated with improved drought tolerance (Jäger et al., 2011). In 

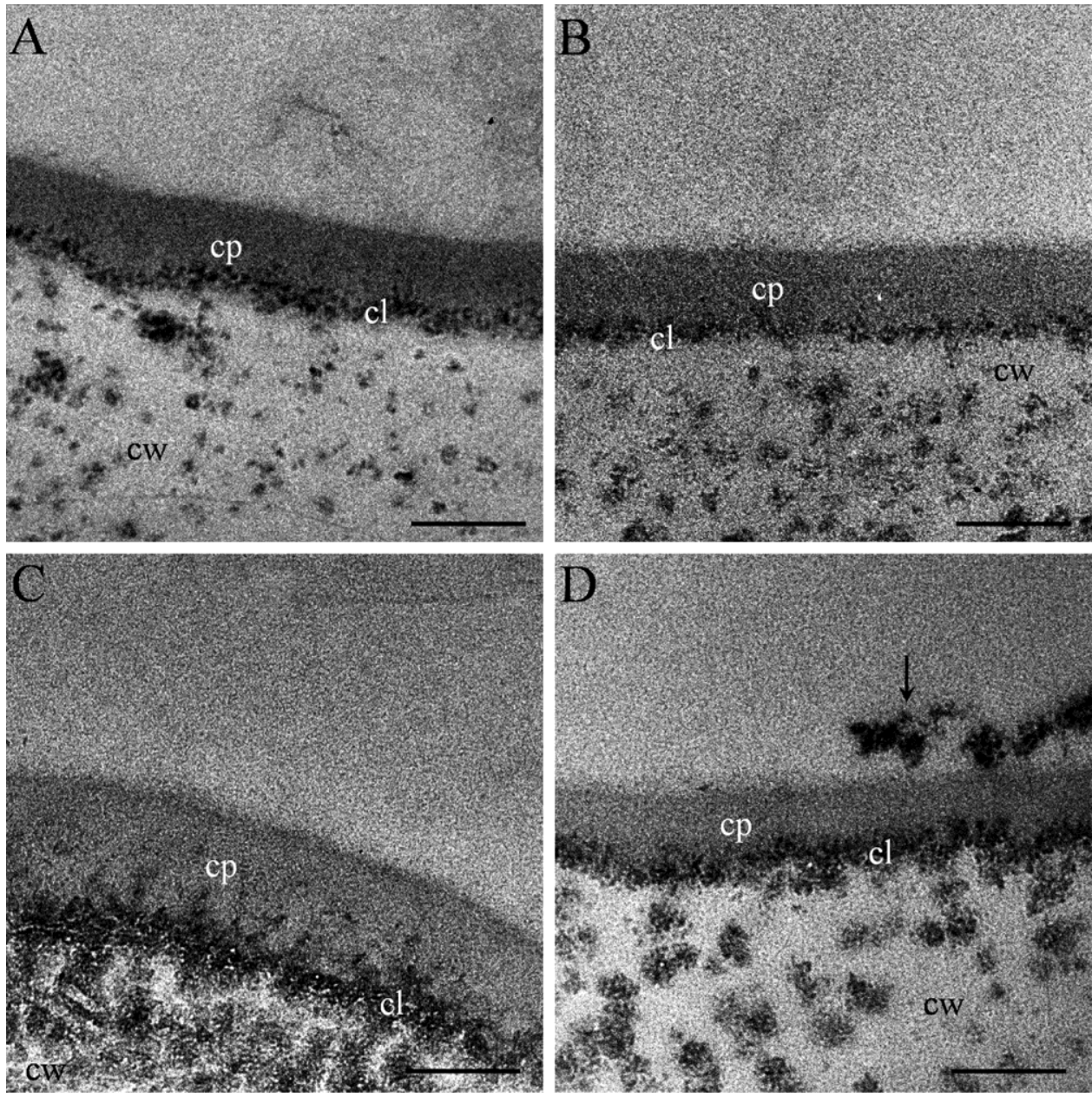

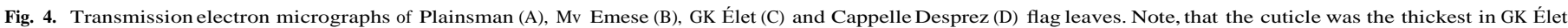
(C) and the thinnest in Cappelle Desprez (D). cl: cuticle layer; cp: cuticle proper; cw: cell wall; arrow: epicuticular waxes. Bars represent 150 nm.

potato lines silenced for an ortholog of the Arabidopsis ABH1/CBP80 gene, more compact cuticular ultrastructure was observed, correlating with a higher rate of water retention of leaves (Pieczynski et al., 2013).

In order to find ultrastructural differences in the epidermal tissues potentially corresponding to drought hardiness, transmission electron microscopy was used on cross sections of leaf samples from the wheat cultivars studied. The structures observed in these micrographs represent the cuticle without the majority of epicuticular waxes, which was removed during sample preparation. Therefore, in our discussion below, cuticular thickness refers to cuticular layer plus cuticle proper, excluding epicuticular waxes.

An unexpected variability of cuticular thickness was found among the wheat cultivars. The drought-sensitive Cappelle Desprez was found to be harboring especially thin cuticles on flag leaves (see Fig. 5 and Supplementary data 1). This was in contrast to the thicker cuticles of the other cultivars, including GK Élet, which showed a low level of drought tolerance. We assume that in GK Élet, traits other than epidermal traits may make a stronger contribution to drought susceptibility. Differences in stress-associated physiological responses of the same wheat varieties have indeed been revealed (Gallé et al., 2009; Guóth et al., 2009; Deák et al., 2011).

The observed substantial variation of leaf cuticular thickness prompted us to look for differences in residual transpiration among the wheat cultivars. Dark-adapted water loss has often been used as an indicator of residual transpiration (e.g. Kosma et al., 2009;
González and Ayerbe, 2010), although incomplete closure of stomata in the dark may obviously have the potential to distort results. Applying this method to flag leaves of the selected wheat cultivars, a higher water loss rate of Cappelle Desprez was observed in comparison with the other cultivars (Fig. 8). This corresponded well with the thin cuticle of this cultivar (Fig. 5; Supplementary data 1). In addition to the direct effects of epidermal traits on water balance, the permeability of the cuticle has also been shown to affect stress defenses through influencing the hormonal response. Wang et al. (2011) has reported that osmotic stress-induced ABA biosynthesis and osmotic stress tolerance were impaired in cutin mutants of Arabidopsis. This implies the possibility that the thin and permeable cuticle of Cappelle Desprez and the low level of stress defense in this cultivar (see below) are not just parallel traits formed during evolution, but might also have a functional connection.

In the case of Arabidopsis, drought stress promoted thickening of the cuticle (Kosma et al., 2009), indicating accelerated biosynthesis of cuticular components. This was paralleled with an increase of drought tolerance and a decrease of residual transpiration. In wheat however we did not find any structural changes of leaf cuticles in response to water deprivation after anthesis, as observed by TEM, despite repeated exposure of the plants to drought conditions (Supplementary data 1 ).

Conflicting data on a role of epicuticular waxes in drought tolerance of cereals have been reported. Gonzalez and Ayerbe (2010) have found a positive correlation between grain yield and epicuticular wax load of barley. In their study, wax coverage increased 

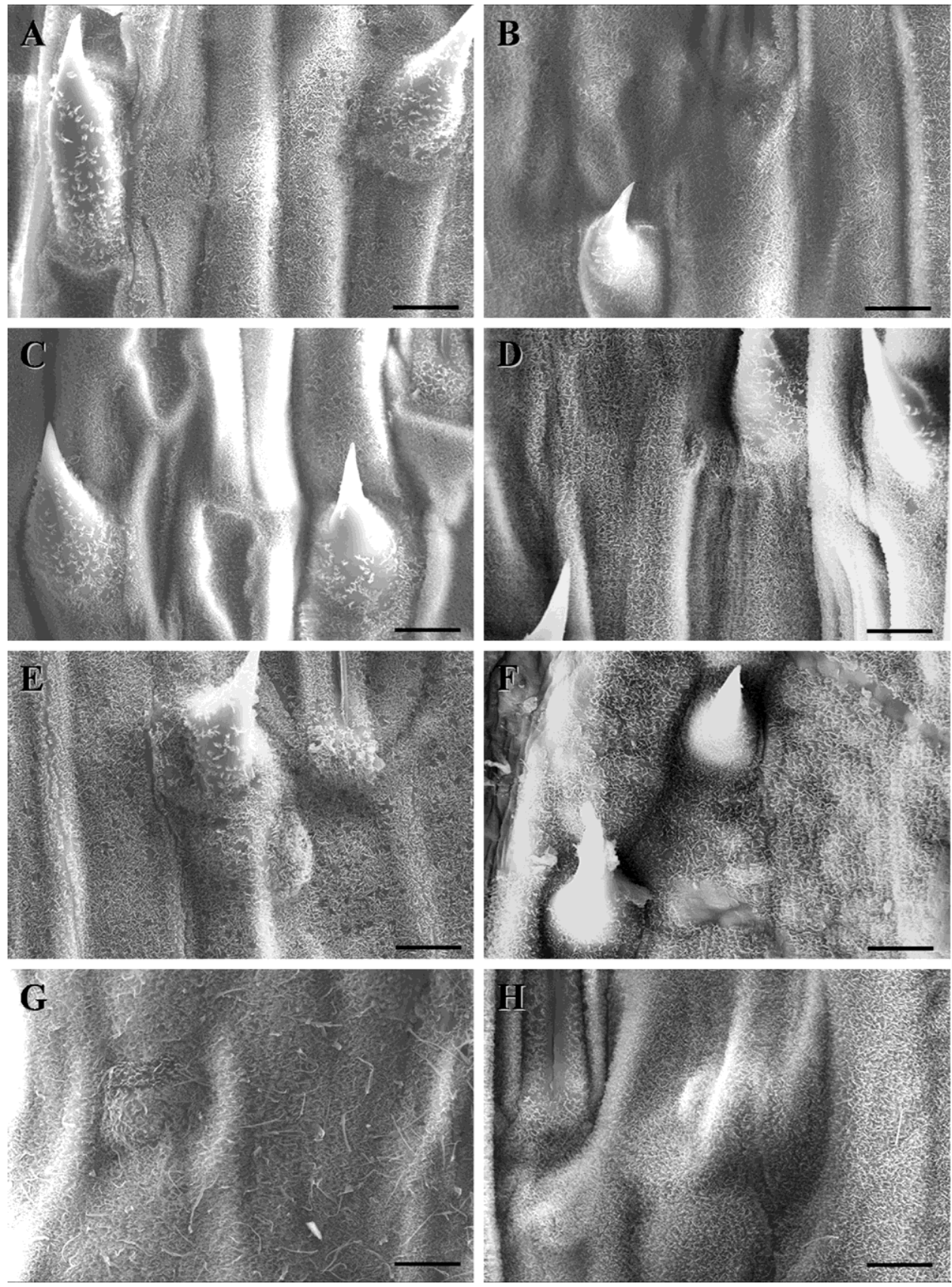

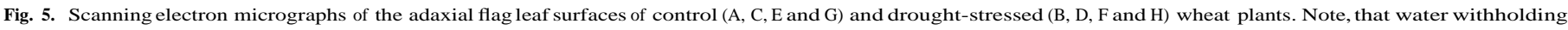
had no effect on wax deposition. Bar $=10 \mathrm{Lm}$.

upon terminal drought stress treatment and this was associated with drought tolerance. On the other hand, the significance of high epicuticular wax load in residual transpiration and drought tolerance has been disputed by others (Larson and Svenningson, 1986; Merah et al., 2000). Glaucousness appeared to confer an advantage to near isogenic wheat lines under rain-fed conditions, rather than in very dry environments (Johnson et al., 1983). An influence of excess amounts of epicuticular waxes on water permeance of the cuticle and on yield under water stress therefore has not been established conclusively in cereals. According to scanning electron microscopy in our studies, drought treatment triggered accumulation of epicuticular wax crystals on the abaxial leaf surfaces in all cultivars. Interestingly, the most drought-sensitive cultivar (Cappelle Desprez) exhibited the most dense filamentous epicuticular wax matrix in comparison with the other genotypes. In our drought stress regime, waxes were deposited predominantly during the second cycle of water deprivation (data not shown). The presence, density, or drought-induced formation of epicuticular wax crystals showed no obvious correlation with drought tolerance. Our data therefore do not support a crucial role for epicuticular wax deposition in drought tolerance of wheat. Morphological differences of the wax structures were evident among the leaf surfaces (see Figs. 5 and 6). Mv Emese leaves displayed platelet-like wax crystals on both leaf surfaces, while the other genotypes held platelet-like and tubular wax depositions on the adaxial and abaxial surfaces, respectively. The morphology of the tubular structures in Cappelle Desprez differed from that of the other cultivars, as they formed a dense interwoven matrix. This variability leads us to speculate that wax compositions on the leaf surfaces may differ. Some variability of epicuticular waxes in Triticeae has already 

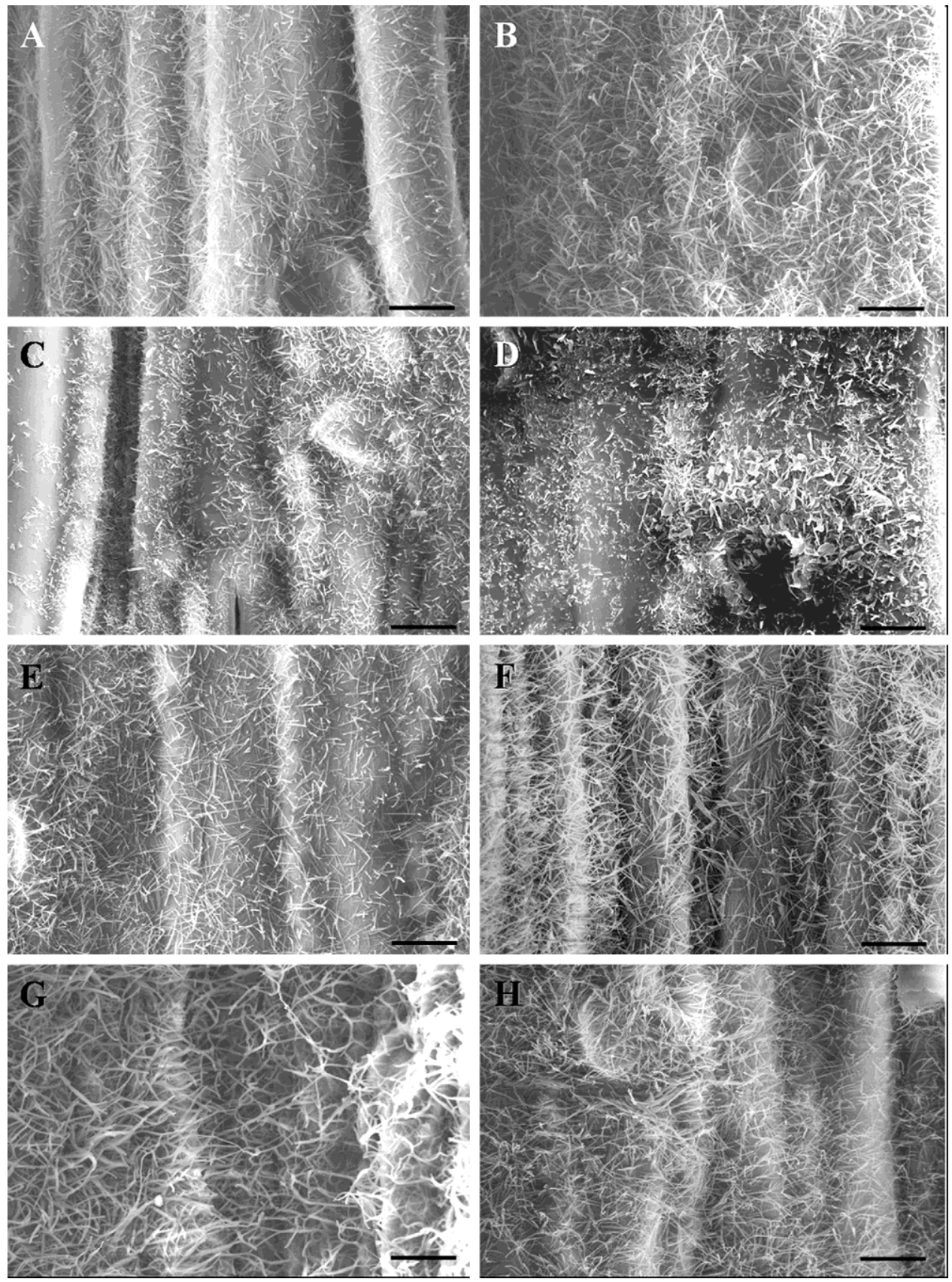

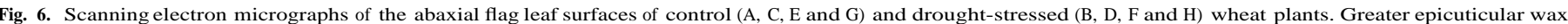
loads are evident on the drought treated samples. Bar $=10 \mathrm{fm}$

been described (Tulloch et al., 1980). More recently Adamski et al. (2013) have noticed tubular/rod-shaped wax structures occurring predominantly on the abaxial leaf surface of a wheat variety.

In addition to epidermal structures, other micromorphological features of leaves from drought-stressed plants were also investigated in order to reveal any bearing on the different drought hardiness of the cultivars. Loss of cellular integrity can be a consequence of redox imbalance, caused by oxidative stress in drought (Mittler, 2002). Structures of chloroplasts, mitochondria, and plasma membranes may be affected by the reactive oxygen species (ROS) produced. The chloroplast, as a major source of ROS generation, may be a prime target for the excess oxidative agents generated during exposure to drought (Gill and Tuteja, 2010). Vacuolized chloroplasts with thylakoidal membrane distortions in mesophyll cells of sensitive apple (Malus hupehensis) leaves were described by Wang et al. (2012a,b). Drought stressinduced destruction of chloroplast structures was also found in female poplar (Zhang et al., 2010). In our experiments, rapid or severe loss of cellular integrity was a hallmark of drought sensitivity. Light microscopy revealed structural damage of chloroplasts predominantly in the sensitive cultivars (Fig. 7). Electrolyte leakage measurements supported this observation. An early increase of conductivity triggered by the first drought treatment occurred in Cappelle Desprez only (Fig. 9). During the second cycle of water stress, conductivity in samples of Cappelle Desprez did not increase further, presumably because a fraction of vulnerable cells had already been disrupted. At the same time, however, an outstanding peak of conductivity was observed in the other sensitive genotype (GK Élet), while the best performing Plainsman V showed the lowest level of cellular injury throughout the experiment. A correlation 

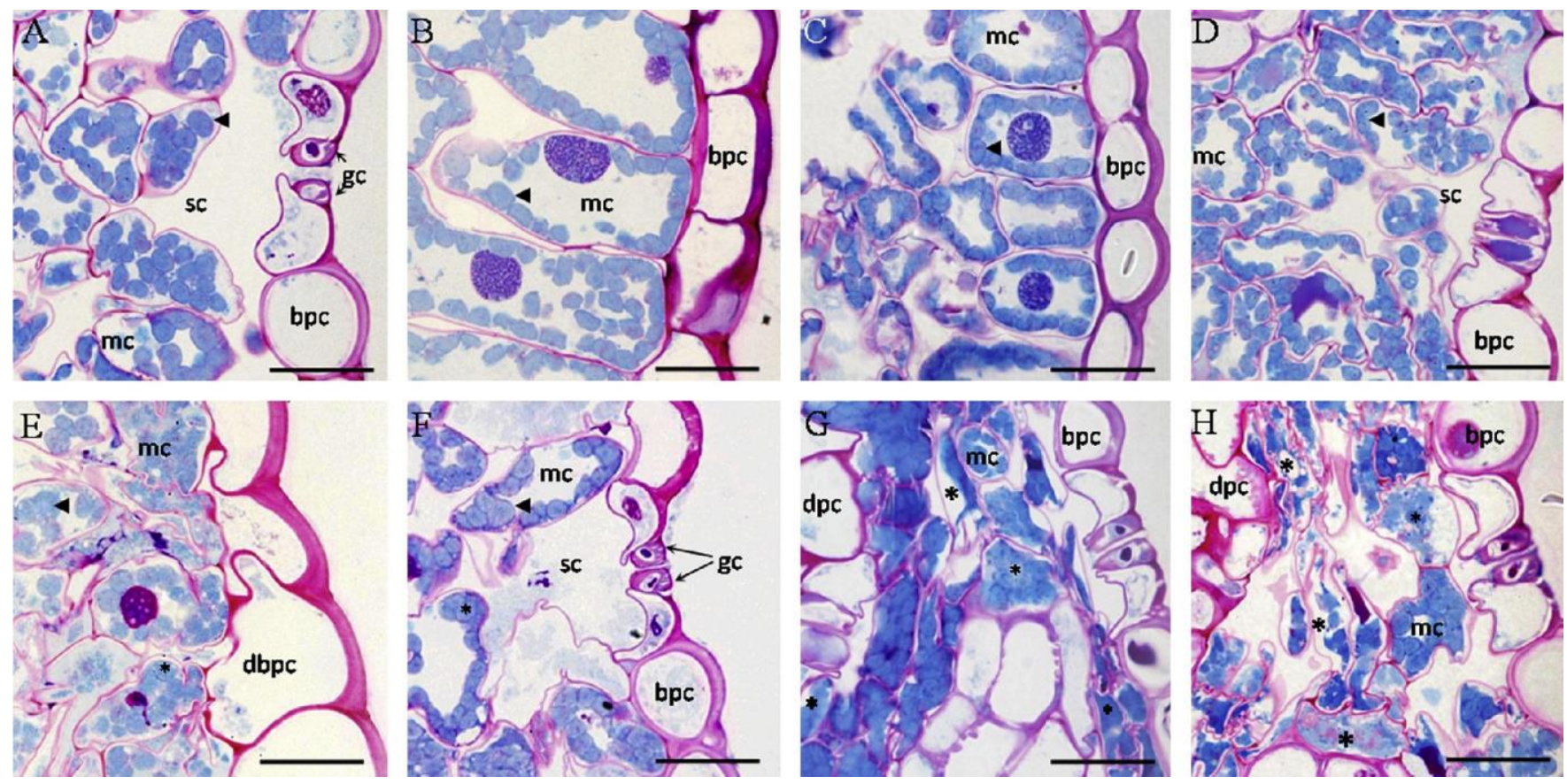

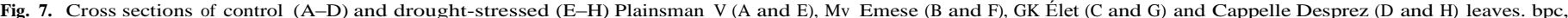

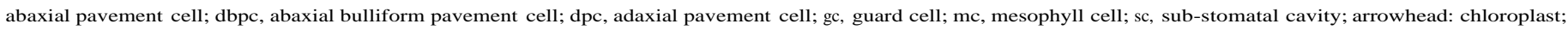
asterisk: mesophyll cell with degenerating chloroplasts. Bars represent $20 \mathrm{p} . \mathrm{m}$.

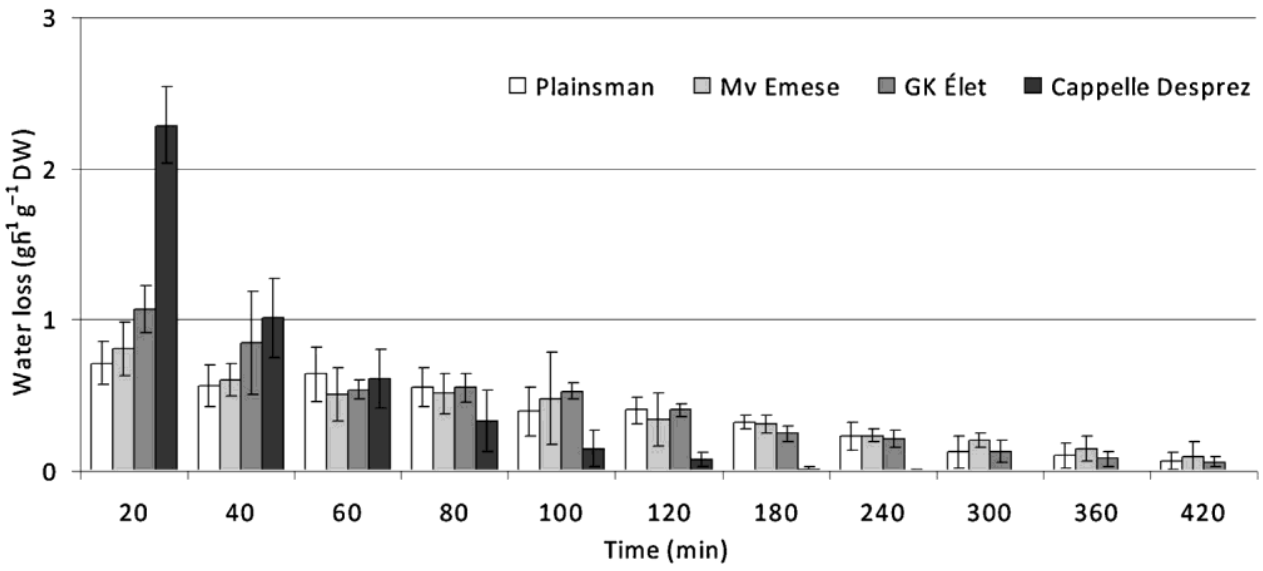

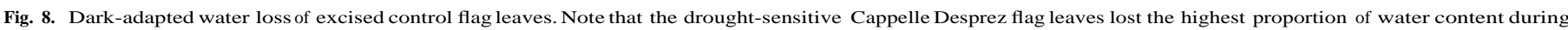
the first $20 \mathrm{~min}$ in comparison with the other genotypes.

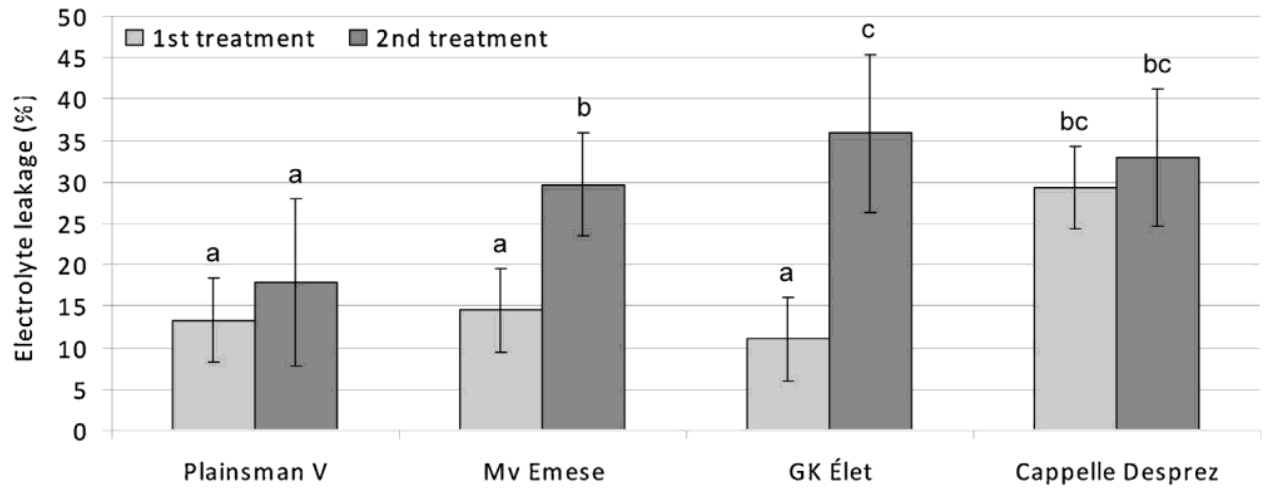

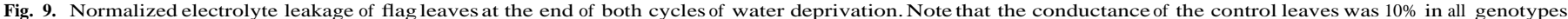

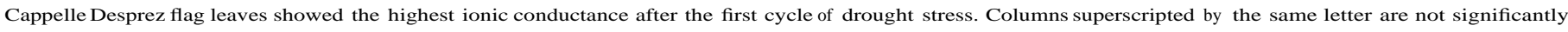
different minimum at the $\mathrm{P} \leq 0.05$ level of probability. 
between drought sensitivity and cellular injuries in wheat varieties has also been noted by Grigorova et al. (2012). In their report, better preservation of organelles was described in a drought-tolerant wheat variety compared to a sensitive cultivar upon water shortage or combined water and heat stress. In this study, observations of cellular integrity by microscopy and ion leakage measurements were complemented by RWC determination. RWC values of the varieties were comparable after the first treatment, except for Cappelle Desprez (sensitive), which displayed a significant drop of leaf water content already by this time. The second drought cycle clearly differentiated sensitive and tolerant genotypes, with tolerant varieties (especially Plainsman V) keeping higher RWC values by the end of the second drying episode (Table 1). Therefore, early or more severe cellular damage and reduced water content of leaves under water stress discriminated drought-sensitive wheat genotypes, with the full spectrum of differences becoming evident only after cyclic water limited conditions.

This finding is consistent with the interpretation that tolerant plants received "drought immunization" during the first water stress, while in sensitive plants this did not happen. This issue however was not addressed at the design of the experiments, therefore our experimental setup did not let draw a firm conclusion in this direction. It is noteworthy at the same time that Khanna-Chopra and Selote (2007) found lower membrane injury as a sign of the drought-acclimation response appearing in a tolerant, but not in a susceptible wheat genotype.

Regarding the contrasting behavior of cultivars, our results fit together well with data of Gallé et al. (2009), who investigated the same set of wheat varieties. High glutathione S-transferase and glutathione oxidase activities were found exclusively in flag leaves of Plainsman V around anthesis. These enzymes are parts of the antioxidant machinery which, among other roles, participates in maintaining membrane integrity in osmotically stressed plants (Dixon et al., 2002). This may potentially explain the resistance of Plainsman V leaf tissues against the detrimental cellular consequences of osmotic stress in drought. Clues about the resistance of Mv. Emese against cellular damage remain to be elucidated, with components of the antioxidant defense system being prime candidates for this role.

Our results strengthen the view that contributions of numerous factors result in the complex phenotype of drought tolerance in wheat (Fleury et al., 2010). Flag leaf area, stomatal density and cuticular thickness are proposed here as relevant factors, while a contribution of epicuticular wax accumulation to tolerance was not substantiated. Structural damage to the chloroplasts, electrolyte leakage and a decline of leaf RWC in water stress were found as indicators of sensitivity, with unambiguous appearance only after repeated exposures to water limitation. Further studies are needed to gain a more complete picture of the physiological processes underlying differences in drought responses of the wheat cultivars studied. It is also important to emphasize that our experiments yielded data concerning only a small set of cultivars. The results obtained therefore obviously need reinforcement by investigating a larger set of wheat genotypes.

Multifactorial determination of plant defenses under stress has been described as complex suites of layered traits by Juenger (2013). The involvement of multiple molecular defense mechanisms in drought tolerance has been also proposed by Chaves and Oliveira (2004). They highlighted the importance of membrane stability of the chloroplast, which is in good agreement with the results presented here.

\section{Acknowledgements}

The authors thank to E. Kapral Bék, E. Gondos and A. Miskó for their excellent technical assistance. The work was supported by the TÁMOP 4.2.1/B-09/1/KMR/-2010-0005, NKTH-OTKA CK80211, NKTH-OTKA CK80274 grants from the National Development Agency of Hungary and the 00044/11 Bolyai János Research Scholarship of the Hungarian Academy of Sciences. C. Deák was supported by the Doctoral Council of Life Sciences of Corvinus University of Budapest.

\section{Appendix A. Supplementary data}

Supplementary material related to this article can be found, in the online version, at http://dx.doi.org/10.1016/j.jplph. 2014.04.013.

\section{References}

Adamski NM, Bush MS, Simmonds J, Turner AS, Mugford SG, Jones A, Findlay K, Pedentchouk N, von Wettstein-Knowles P, Uauy C. The inhibitor of wax 1 locus (Iw1) prevents formation of $\{3$ - and $\mathrm{OH}$-\{3-diketones in wheat cuticula waxes and maps to a sub-cM interval on chromosome arm 2BS. Plant J 2013. http://dx.doi.org/10.1111/tpj.12185.

Araus JL, Slafer GA, Reynolds MP, Royo C. Plant breeding and drought in C3 cereals: what should we breed for? Ann Bot 2002;89:925-40

Biswal AK, Kohli A. Cereal flag leaf adaptations for grain yield under drought: knowledge status and gaps. Mol Breed 2013;31:749-66.

Chaves MM, Oliveira MM. Mechanisms underlying plant resilience to water deficits: prospects for water-saving agriculture. J Exp Bot 2004;55:2365-84

Clarke JM, Richards RA, Condon AG. Effect of drought stress on residual transpiration and its relationship with water use of wheat. Can J Plant Sci 1991;71:695-702.

Cruz de Carvalho MH. Drought stress and reactive oxygen species: production, scavenging and signaling. Plant Signal Behav 2008;3:156-65.

Deák C, Jäger K, Fábián A, Nagy V, Albert Z, Miskó A, Barnabás B, Papp I. Investigation of physiological responses and leaf morphological traits of wheat genotypes with contrasting drought stress tolerance. Acta Biol Szeged 2011;55:69-71.

Dixon DP, Lapthorn A, Edwards R. Plant glutathione transferases. Genome Biol 2002;3, reviews3004-reviews3004.10.

Doheny-Adams T, Hunt L, Franks PJ, Beerling DJ, Gray JE. Genetic manipulation of stomatal density influences stomatal size, plant growth and tolerance to restricted water supply across a growth carbon dioxide gradient. Philos Trans R Soc Lond B: Biol Sci 2012;367:547-55

Fábián A, Jäger K, Rakszegi M, Barnabás B. Embryo and endosperm development in wheat (Triticum aestivum L.) kernels subjected to drought stress. Plant Cell Rep 2011;30:551-63.

Fleury D, Jefferies S, Kuchel H, Langridge P. Genetic and genomic tools to improve drought tolerance in wheat. J Exp Bot 2010;61:3211-22.

Gallé Á, Csiszár J, Secenji M, Guóth A, Cseuz L, Tari I, Györgyey J, Erdei L. Glutathione transferase activity and expression patterns during grain filling in flag leaves of wheat genotypes differing in drought tolerance: response to water deficit. J Plant Physiol 2009; 166:1878-91

Gill SS, Tuteja N. Reactive oxygen species and antioxidant machinery in abiotic stres tolerance in crop plants. Plant Physiol Biochem 2010;48:909-30.

González A, Ayerbe L. Effect of terminal water stress on leaf epicuticular wax load, residual transpiration and grain yield in barley. Euphytica 2010;172:341-9.

Grigorova B, Vassileva V, Klimchuk D, Vaseva I, Demirevska K, Feller U. Drought, high temperature, and their combination affect ultrastructure of chloroplasts and mitochondria in wheat (Triticum aestivum L.) leaves. J Plant Interact 2012;7:204-13.

Guóth A, Tari I, Gallé Á, Csiszár J, Pécsváradi A, Cseuz L, Erdei L. Comparison of the drought stress responses of tolerant and sensitive wheat cultivars during grain filling: changes in flag leaf photosynthetic activity. ABA levels, and grain yield. J Plant Growth Regul 2009;28:167-76.

Izanloo A, Condon AG, Langridge P, Tester M, Schnurbusch T. Different mechanisms of adaptation to cyclic water stress in two South Australian bread wheat cultivars. J Exp Bot 2008;59:3327-46

Jäger K, Fábián A, Tompa G, Deák C, Höhn M, Olmedilla A, Barnabás B, Papp I. New phenotypes of the drought-tolerant cbp20 Arabidopsis thaliana mutant have changed epidermal morphology. Plant Biol 2011;13:78-84.

Johnson DA, Richards RA, Turner MC. Yield, water relations, gas exchange and surface reflectances of near-isogenic wheat lines differing in glaucousness. Crop Sci $1983 ; 23: 318-25$.

Juenger TE. Natural variation and genetic constraints on drought tolerance. Curr Opin Plant Biol 2013;16:274-81

Khanna-Chopra R, Selote DS. Acclimation to drought stress generates oxidative stress tolerance in drought-resistant than -susceptible wheat cultivar under field conditions. Environ Exp Bot 2007;60:276-83.

Kosma DK, Bourdenx B, Bernard A, Parsons EP, Lü S, Joubès J, Jenks MA. The impact of water deficiency on leaf cuticle lipids of Arabidopsis. Plant Physiol 2009; 151:1918-29.

Larson S, Svenningson M. Cuticular transpiration and epicutiular lipids of primary leaves of barley (Hordeum vulgare L.). Physiol Plant 1986;68:13-9. 
1266

K. Jäger et al. / Journal of Plant Physiology 171 (2014) 1256-1266

Merah O, Deléens E, Souyris I, Monneveux P. Effect of glaucousness on carbon isotope discrimination and grain yield in durum wheat. J Agron Crop Sci 2000; $185: 259-65$.

Mittler R. Oxidative stress, antioxidants and stress tolerance. Trends Plant Sci 2002; 7:405-10.

Murray MB, Cape JN, Fowler D. Quantification of frost damage in plant tissues by rates of electrolyte leakage. New Phytol 1989;113:307-11.

Percival J. The wheat plant. A monograph. London, United Kingdom: Duckworth and Co; 1921.

Pieczynski M, Marczewski W, Hennig J, Dolata J, Bielewitz D, Piontek P, Wyrzykowska A, Krusiewicz D, Strzelczyk-Zyta D, Konopka-Postupolska D, Krzeslowska M, Jarmolowsky A, Szweykowska-Kulinska Z. Down-regulation of CBP80 gene expression as a strategy to engineer a drought-tolerant potato. Plant Biotechnol J 2013;11:459-69.

Rawson HM, Clarke JM. Nocturnal transpiration in wheat. Aust J Plant Physiol 1988; 15:397-406.

Riederer M, Schreiber L. Protecting against water loss: analysis of the barrier properties of plant cuticles. J Exp Bot 2001;52:2023-32.

Rustic Z, Jenks MA. Leaf cuticle and water loss in maize lines differing in dehydration avoidance. J Plant Physiol 2002;59:645-51.

Salisbury EJ. On the causes and ecological significance of stomatal frequency, with special reference to the woodland flora. Pills Trans R Soc Lond B: Biol Sci $1927 ; 16: 1-65$.

Sečenji M, Hided É, Bebes A, Györgyey J. Transcriptional differences in gene families of the ascorbate-glutathione cycle in wheat during mild water deficit. Plant Cell Rep 2010;29:37-50.

Sirichandra C, Wasilewska A, Vlad F, Valon C, Lung L. The guard cell as a single-cell model towards understanding drought tolerance and abscisic acid action. J Exp Bot 2009;60:1439-63.
Spurn AR. A low viscosity epoxy embedding medium for electron microscopy. J Ultrastruct Res 1969;26:31-43.

Szủcs A, Jäger K, Jurca ME, Fábián A, Bottka S, Zvara Á, Barnabás B, Fehér A. Histological and microarray analysis of the direct effect of water shortage alone or combined with heat on early grain development in wheat (Triticum aestivum). Physiol Plant 2010;140:174-88.

Tischner T, Kőszegi B, Veisz O. Climatic programmes used in the Martinvásárphytotron most frequently in recent years. Act Agron Hung 1997;45: 85-104.

Tulloch AP, Baum BR, Hoffman LL. A survey of epicuticular waxes among genera of Triticeae. 2. Chemistry. Can J Bot 1980;58:2602-15.

Wang ZY, Xiong L, Li W, Zhu JK, Zhu J. The plant cuticle is required for osmotic stress regulation of abscisic acid biosynthesis and osmotic stress tolerance in Arabidopsis. Plant Cell 2011;23:1971-84.

Wang S, Liang D, Li C, Mao Y, Ma F, Shut H. Influence of drought stress on the cellular ultrastructure and antioxidant system in leaves of drought-tolerant and drought-sensitive apple rootstocks. Plant Physio Biochem 2012a;51: $81-9$.

Wang Y, Wan L, Chang L, Chang Z, Chang H, Guan R, Chou S, Huang R. An ethylene response factor OsWR1 responsive to drought stress transcriptionally activates wax synthesis related genes and increases wax production in rice. Plant Mol Biol 2012b;78:275-88.

Zadoks JC, Chang TT, Konzak CF. A decimal code for growth stages of cereals. Weed Res $1974 ; 14: 415-21$.

Chang S, Chen F, Peng S, Ma W, Korpelainen H, Li C. Comparative physiological, ultrastructural and proteomic analyses reveal sexual differences in the responses of Populus cathayana under drought stress. Proteomics 2010;10: 2661-77. 